\title{
The Optics and Optimal Control Theory Interpretation of the Parametric Resonance
}

\author{
Nikolay Nikolaevitch Schitov \\ All-Russian Dukhov Automatics Research Institute, Moscow, Russia
}

Email address:

nschitov@mail.ru

\section{To cite this article:}

Nikolay Nikolaevitch Schitov. The Optics and Optimal Control Theory Interpretation of the Parametric Resonance. American Journal of Physics and Applications. Vol. 7, No. 3, 2019, pp. 73-83. doi: 10.11648/j.ajpa.20190703.13

Received: April 22, 2019; Accepted: June 4, 2019; Published: June 26, 2019

\begin{abstract}
The aim of the article is the elaboration of parametric resonance theory at piecewise constant frequency modulation. The investigation is based on the analogy with optics and optimal control theory (OCT) application. The exact expressions of oscillation frequency, gain/damping coefficients, dependencies of these coefficients on the modulation depth, duty ratio and initial phase are derived. First of all, the results obtained on the basis of the energy behavior analysis (at the conjunction conditions execution) in frictionless systems are presented. The well-known parametric resonance triggering condition is revised and adjusted. The heuristic feedback introduction (based on the energy behavior analysis) in the oscillation equation permits one to prove that the frequency modulation satisfying the parametric resonance condition is not necessary and sufficient condition of the oscillations unlimited increase. Their damping/shaking up formally corresponds by the frequency and duty ratio to the condition of the equality of optical paths to the quarter-wavelength characteristic of the interference filter or mirror. The unity of space-time coordinates shows itself in this specific form of the optical-mechanical analogy due to the general Hill's equation description. It is marked that this equation theory underlies most of metamaterials advantages because all transport phenomena imply different wave - electromagnetic, acoustic, spin etc. propagation one way or another. The question about control uniqueness arises that is modulating frequency, duty ratio and signature sign uniqueness. Another question of characteristic index extremum at different controls is tightly bound with the former. The answers to these questions are obtained on the basis of OCT. The similarity of the optimal control problem solution and the one obtained at the heuristic feedback introduction through fundamental solutions product permits one to introduced the new form named general or mixed Hamiltonian along with the ordinary and OCT Hamiltonians. Besides this mixed Hamiltonian equality to zero together with the Wronskian constancy (almost everywhere) is the useful analogous in form to the Liouville's theorem equation. The nonlinearity accounting using the OCT formalism is described too.
\end{abstract}

Keywords: Parametric Resonance, Optimal Control Theory, Hill's Equation, Bragg Condition, Optical-Mechanical Analogy

\section{Introduction}

The parametric resonance theory of single degree-offreedom oscillating systems is based on the Hill's equation analysis [1]:

$$
\ddot{x}=-\omega^{2}(1+w \cdot a(t)) \cdot x, w<<1, a(t+T)=a(t)
$$

The main conclusions of this analysis deal only with the solution dependence on the modulating function frequency (or wave vector for space oscillations) but don't determine this function kind. For example, the equality of modulation frequency to doubled eigenmodes frequency (analogous to the Bragg condition in optics) isn't completed by any other function's characteristics such as duty ratio, modulation depth (rate) etc. At this even the parametric resonance condition in the form of the modulating function frequency equality to doubled - "Bragg's" - is not always expressed explicitly (see, for instance, [2]).

On assumption of the modulation depth smallness $(w<<1)$ the condition of parametric resonance onset is obtained for the case when the deviation $\varepsilon$ from the fundamental frequency $\omega_{0}$ satisfies [3]:

$$
-\frac{w \cdot \omega_{0}}{2}<\varepsilon<\frac{w \cdot \omega_{0}}{2}
$$


The relation (2) is derived though in the initial assumption that "...parametric resonance occurs if the modulating frequency $\omega(t)$ is near the doubled frequency $\omega_{0}$ " ([3], p. 83). That's why the obtained result cannot serve as a strict proof of the parametric resonance onset just at doubled modulating frequency and, moreover, doesn't give any clear physical interpretation of the effect.

Another more rigorous parametric resonance analysis though executed as well on assumption of modulation depth smallness is presented in a study [4]. It is based on the geometric interpretation of equation (1) properties - selfadjoint differential form. At that the constancy and equality of Wronskian $(W(\mathrm{x}))$ to unity is interpreted as the equality of the matrix $A$ (linear plane self-mapping) determinant to unity - reflection upon the period conserving the area:

$A=\left(\begin{array}{ll}a_{11} & a_{12} \\ a_{21} & a_{22}\end{array}\right)=\left(\begin{array}{cc}x_{1}(T) & x_{2}(T) \\ x_{1}{ }^{\prime}(T) & x_{2}{ }^{\prime}(T)\end{array}\right) \Rightarrow \operatorname{det} A=|A| \equiv W(T)=1$,

where $x_{1}(t), x_{2}(t)$ - fundamental solutions of (1), so that $x_{1}(0)=1, x_{1}{ }^{\prime}(0)=0$ and $x_{2}(0)=0, x_{2}{ }^{\prime}(0)=1$. The spur of this matrix determines whether this reflection is the turning and that's why steady or hyperbolic turning - that is unsteady. The transition boundary is determined by the equality $|\operatorname{tr} A|=2$. From the Hill's equation theory viewpoint the matrix spur is the determining parameter of the characteristic equation at the condition of Wronskian equality to unity [1]:

$$
\begin{gathered}
\operatorname{Det}\left(a_{p, q}-s \cdot e_{p, q}\right)=0 \Rightarrow \\
s^{2}-\left[x_{1}(T)+x_{2}{ }^{\prime}(T)\right] s+W(T)=s^{2}-\operatorname{tr} A \cdot s+1=0,
\end{gathered}
$$

And its value surpassing 2 means the existence of this equation real roots. The parametric resonance condition is obtained at the replacement of exact Hill's equation solutions in the matrix $A$ on to harmonic ones (at the modulation depth tending to zero) and equalizing the spur to 2 .

$$
x_{1}(t) \cong \cos (\omega \cdot t), x_{2}(t) \cong \frac{1}{\omega} \sin (\omega \cdot t) \Rightarrow \operatorname{tr} A=2 \cos (2 \pi \omega)=2 .
$$

This implies that the unsteady systems set may really approach the axe $\omega$ (on the $w, \omega$ parameters plane) only in the points $\omega=\mathrm{k} / 2, \mathrm{k}=1,2, \ldots$, where the spur is equal to 2 . The unsteadiness or parametric resonance is interpreted as the unlimited oscillation amplitude and speed rise (of course in the linear approximation and friction absence) at the argument going to infinity. It follows from the fact that in the general case the characteristic equation solution at $\operatorname{tr} \mathrm{A}>2$ has one root bigger modulo 1 (the second correspondingly smaller) that is the characteristic index is positive so that one of fundamental solutions is exponentially growing. Thus, the parametric resonance theory (presented in manuals) is reduced in fact to the determination of its onset condition at the modulation depth smallness without its thorough explanation. Whereas calculations of the true oscillation frequency (differing from the eigenmode one), characteristic indexes (gain/damping coefficients), their dependence on the modulation depth and duty factor (control - in OCT terms [5]) are absent even for the simplest case of piecewise constant frequency modulation. (Although this case is never realized due to the fundamental reason: the instantaneous frequency of an unsteady process is the derivative of the corresponding analytical signal and is continuously differentiable [6]).

The exact description of the phenomenon needs the energy balance analysis. It's well-known that a swing may be either shaken up or braked at the periodic length changing. However, the control changes at that are apriori unclear. In other words, it's necessary to investigate what will be the control frequency and duty factor in both cases and calculate all other process characteristics.

\section{The Energy Behavior at Frequency Change}

The energy balance analysis is made in many articles and monographs concerning different oscillating systems including the mentioned above swing [7], electric vibration LRC contour [8], torsional vibration spring oscillator [9-12]. The energy balance condition consists of the equality to zero the sum of losses (due to a friction for instance) and useful (that is needed to change periodically the control parameter) works. So main efforts were made to correctly calculate these works in particular cases. Neglecting these particular peculiarities one may note some general aspects.

The oscillating system with time-depending frequency is the Hamilton's but not autonomic system. It means that the system Hamiltonian may be written in a standard form (the argument is denoted as $x$ for time and space oscillations unity):

$$
\begin{aligned}
& H(x)=\frac{1}{2} \cdot\left(p^{2}(x)+\omega^{2}(x) \cdot y^{2}(x)\right) \neq \text { Const }, \\
& p(x)=y^{\prime}(x)=\frac{\partial H}{\partial p} \Rightarrow p^{\prime}(x)=-\omega^{2}(x) \cdot y(x)=-\frac{\partial H}{\partial y}
\end{aligned}
$$

At the sharp frequency alteration the energy may either change or not. This interesting oscillating system peculiarity expresses explicitly at the conjugation condition realization that is the amplitude and its derivative (oscillation speed) continuity in the point where the frequency value changes. In a "node" that is in a zero amplitude point from the derivatives equality follows the inversely proportionality of amplitudes to corresponding frequencies so that the energy is conserved:

$$
\omega_{1} \cdot Y_{1}=\omega_{2} \cdot Y_{2} \Rightarrow Y_{1} / Y_{2}=\omega_{2} / \omega_{1} \Rightarrow H_{1}=H_{2} \equiv H_{0} .
$$

In a "antinode" that is in a zero derivative point the conjugation conditions at the piecewise frequency change lead to the piecewise energy change too:

$$
Y_{1}=Y_{2} \Rightarrow H_{1} / H_{2}=\left(\omega_{1} / \omega_{2}\right)^{2}, \Delta H=\left(\omega_{1}^{2}-\omega_{2}^{2}\right) \cdot Y^{2}
$$


The oscillation damping is possible if the frequency change - from bigger to smaller - occurs when the amplitude is near an antinode. In the general case the conjugation conditions for the oscillation at the frequency $\omega 1$ of unit amplitude with the oscillation at the frequency $\omega 2$ of amplitude $B$ may be written in the form (compare with the similar conditions in [9]):

$$
\left\{\begin{array}{c}
\operatorname{Cos}(\omega 1 \cdot x)=B \cdot \operatorname{Cos}(\omega 2 \cdot(x+\phi)) \\
\omega 1 \cdot \operatorname{Sin}(\omega 1 \cdot x)=\omega 2 \cdot B \cdot \operatorname{Sin}(\omega 2 \cdot(x+\phi)) \\
B=\operatorname{Cos}(\omega 1 \cdot x) / \operatorname{Cos}(\omega 2 \cdot(x+\phi)) \\
\phi=\frac{1}{\omega 2} \operatorname{arctg}\left[\frac{\omega 1}{\omega 2} \operatorname{tg}(\omega 1 \cdot x)\right]-x
\end{array} \quad,(\omega 1=\omega \cdot \sqrt{1+w}, \omega 2=\omega \cdot \sqrt{1-w})\right.
$$

Although the energy changes absolute values are always equal for both cases their relative values are never equal. For example, if the frequency switching occurs in antinodes the ratio of relative energy increase to relative decrease is equal $(1+w) /(1-w)>0$. It, of course, does not mean that the oscillation shaking up occurs - if switching occurs strictly in the function or its derivative extrema the energy during the period is conserved. In the general case the relative energy increase at the transition to bigger frequency is always greater than the relative decrease of the energy in the opposite case. Such energy behavior explains the oscillation shaking up at the modulating frequency equal to doubled main one and duty factor equal to 2 .

It is clear that if the transition to miner frequency occurs always at $\left(1-R E\left(x_{i}\right)\right) \cdot\left(1+R E 1\left(x_{i+1}\right)<1\right.$ oscillations are damping because the energy decrease will not be compensated by its increase at the transition to the larger one. It is clear too that the whole stop will never occur at this modulation but it is quite possible to approach the phase plane origin arbitrarily near during the finite time. This origin in this case will be the steady focus whereas in an arbitrary case it will be the unsteady focus.

\section{The Feedback Heuristic Introduction}

From the previous results unambiguously follows the possibility of oscillation damping in the case of switching points strict coincidence with oscillation equation's solution singular points. To prove it let us present the solution of the following equation (the first fundamental solution is denoted by the index 1 and the heuristic control - by the index "e"):

$$
\begin{aligned}
& y 1 e^{\prime \prime}(x)+\omega_{0}^{2}\left(1+w \cdot \operatorname{sign}\left(y 1 e(x) \cdot y 1 e^{\prime}(x)\right)\right)^{2} \cdot y 1 e(x)=0, \\
& y 1 e(0)=1, y 1 e^{\prime}(0)=0
\end{aligned}
$$

The solution, control (modulating function) and phase trajectory for $w=0.1, \omega_{0}=0.2 \pi(T=10)$ are presented on figures 1,2 . These curves testify that the phase plane origin is the steady focus in this case. It is clearly seen that the frequency switching occurs just in the points where the solution itself or its derivative (amplitude or speed) changes its sign. At that the first switching occurs in the origin where the amplitude of "undisturbed" oscillations is maximal and the speed is zero and changes its sign.

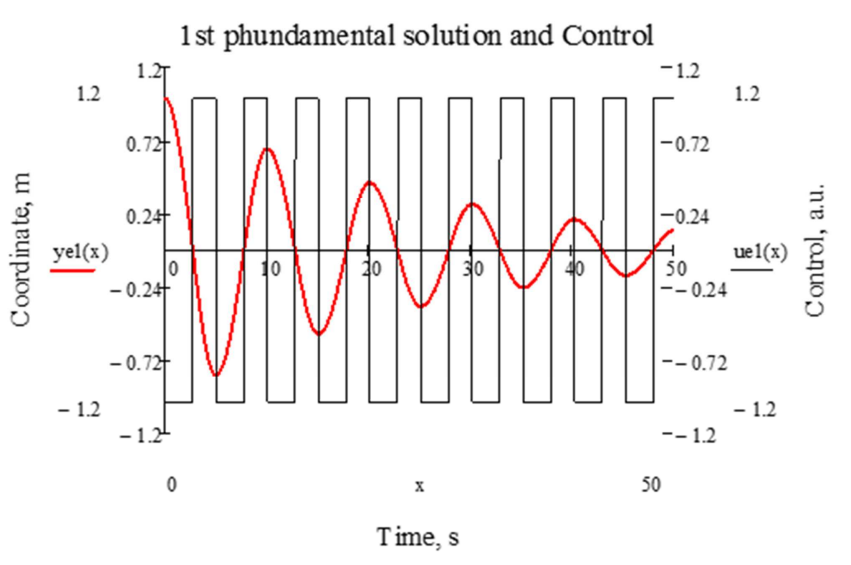

Figure 1. $1^{\text {st }}$ fundamental solution $y l e(x)$ and control ule $(x)$ at the "negative feedback introduction".

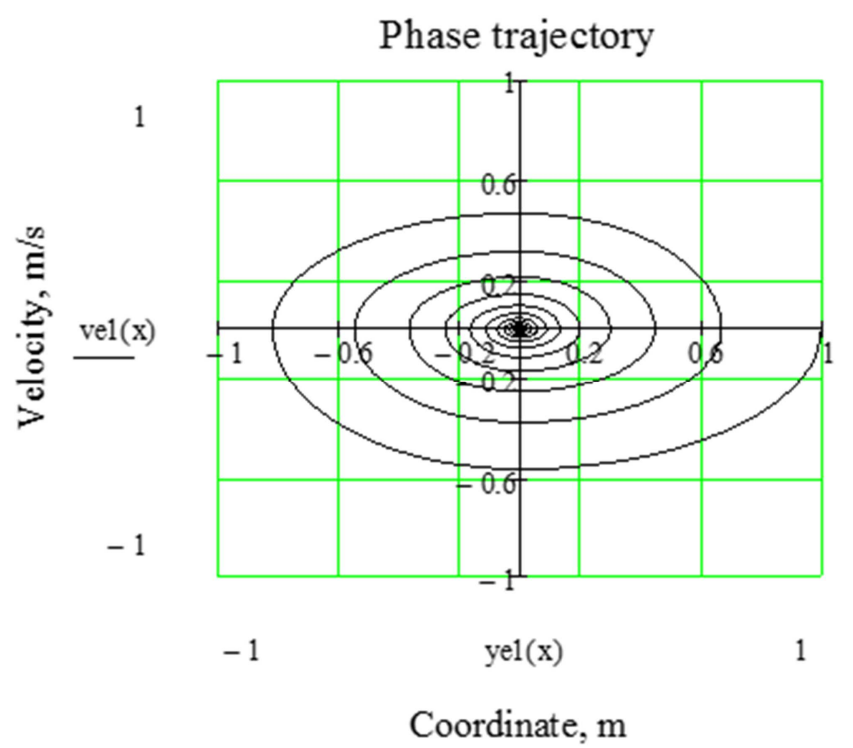

Figure 2. Phase trajectory at the "negative feedback introduction"; vle (x)the $1^{\text {st }}$ fundamental solution derivative (speed).

The sign before the control signature is chosen positive to switch the frequency from high to low in antinodes that is to decrease energy. The inverse switching will occur in nodes where energy change is absent. Thus, the "negative feedback" (if one may say it) is introduced in the equation whereas any driving force is absent. Let us note that for this control the oscillations damping will occur at any initial conditions and any modulation depth. The negative sign 
before the signature at (7) corresponds to the control providing oscillations shaking up independently upon the initial conditions and modulation depth as well whereas the condition (2) may not be executed at that. For instance, at $\mathrm{w}>0.5$ oscillations will shake up or damp depending on the control sign the faster the larger is the modulation depth (to the certain limit - see later). Of course, such a feedback may not be obligatory piecewise but may be continuous. Still the damping will be rather slow (see, for example [7-9]).

To estimate the damping efficiency (during 10 periods) let's introduce two criteria: $K$ and $S$ according to the formula:

$$
K=\int_{0}^{100}\left[(y(x))^{2}+(v(x))^{2}\right] d x, \quad S=\frac{1}{2} \int_{0}^{100}\left[\omega^{2}(x)(y(x))^{2}+(v(x))^{2}\right] d x=\int_{0}^{100} H(x)
$$

The first criterion determines the speed of the phase plane origin approaching, the second equal to the action according to its sense and dimension - the oscillation energy decrease efficiency for the chosen kind of the control. They are equal in this particular case to: $K e=8,943 ; S e=2,447$ (index "e" means "heuristic" as previously).

It seems that the oscillation frequency is equal to the fundamental one and the control's - the Bragg's one. This frequency undoubtedly satisfies the parametric resonance condition, but oscillations nevertheless are damping. The characteristic index may easily be approximately calculated from the conjugation condition on assumption of near equality of the oscillation frequency to the fundamental one. During the half-period the amplitude's decrease will be equal according to (4):

$$
\begin{aligned}
& Y(T) / Y(0)=\omega 1 / \omega 2=(1-w) /(1+w), \\
& Y(T / 2)=Y(0) \cdot \exp (\alpha \cdot T / 2) \Rightarrow \alpha=\frac{2}{T} \ln \left(\frac{1-w}{1+w}\right) \approx-0,04
\end{aligned}
$$

The oscillation frequency specification and correspondingly the characteristic index may be evaluated using the frequency switching condition just precisely in nodes and antinodes. It means that different frequencies oscillation times may be determined from the condition of their equality to quarter-period for corresponding frequencies - equality of optics ways to quarter-wavelength if one use the optic analogy. Let's denote these times $\tau_{+}$and $\tau_{-}$and evaluate the expressions for oscillations frequency $-\omega$, modulating $\omega_{m}$, duty ratio $-D R$ and the characteristic index $\alpha$ :

$$
\begin{aligned}
& \tau_{+}=\frac{1}{4} \cdot \frac{2 \pi}{\omega_{0}(1+w)}=\frac{T}{4(1+w)}, \tau_{-}=\frac{T}{4(1-w)} \Rightarrow \\
& T_{\Sigma}=\tau_{+}+\tau_{-}=\frac{T}{2\left(1-w^{2}\right)} \Rightarrow \omega_{m}=\frac{2 \pi}{T_{\Sigma}}=2 \cdot \omega_{0} \cdot\left(1-w^{2}\right) \equiv 2 \omega \\
& D R=\frac{T_{\Sigma}}{\tau_{+}}=\frac{2}{1-w} ; \alpha=\frac{2 \cdot\left(1-w^{2}\right)}{T} \cdot \ln \left(\frac{1-w}{1+w}\right)
\end{aligned}
$$

The modulation frequency is really equal to the double true oscillation frequency $\omega$ smaller than the fundamental in $\left(1-w^{2}\right)$ times. The duty ratio occurs to be more than 2 . These parameters provide oscillations shaking up/damping (depending on the control sign) independently on the modulation depth. The reduced characteristic index $\mathrm{T} \alpha$ (modified attenuation index) that is divided on the fundamental frequency or multiplied on the fundamental period plot is given on figure 3 (the modulation depth taken variable is denoted as $\xi$ and called "modulation rate"). In the 0 to 0.4 interval the reduced index is almost linear function of the modulation depth: $T \alpha(\xi) \cong 3,56 \cdot \xi$. It's interesting that this dependency has the minimum in the point $\xi \equiv w=0,648$. The reduced characteristic modulo in it is equal to 1.791 that is at period equal to 10 the characteristic index is equal to 0.1791 . Thus, at this frequency modulation method the maximal oscillations increase/decrease is obtained at the strictly determined modulation depth.

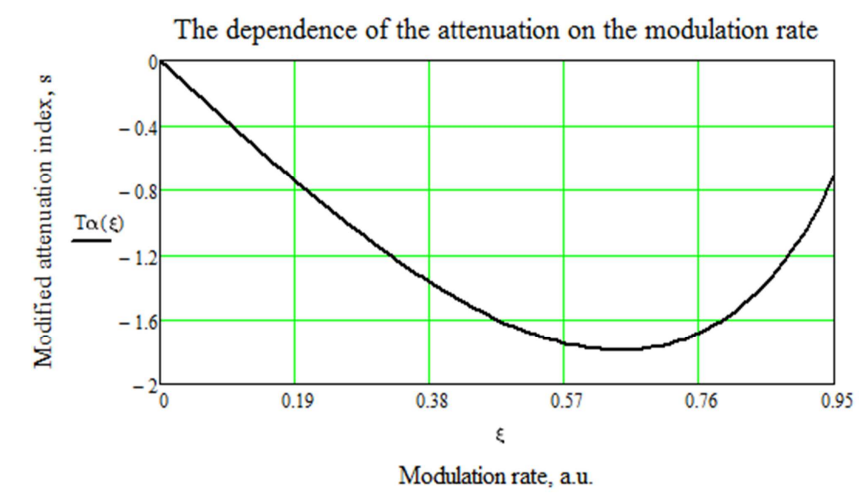

Figure 3. The reduced characteristic index dependency on the modulation depth.

The feedback introduction method considered above is obviously not unique. As a "key" one may take not the solution and its derivative product, but the product of the fundamental solutions. The oscillating systems corresponding to different fundamental solutions are conjugated (not in the sense of the conjugation conditions (6) but in the sense of the belonging to conjugated differential forms). Because the oscillation equation differential form is self-conjugated the difference in the solutions is expressed only in the initial conditions difference. In this case the $2^{\text {nd }}$ order 2 equations system is solved (or 4 equations of the $1^{\text {st }}$ order; index 1 corresponds to the $1^{\text {st }}$ fundamental solution as before, and by letter $s$ we denote solutions at heuristic feedback introduction through fundamental solutions product):

$$
\left\{\begin{array}{c}
y 1 s^{\prime}(x)=v 1 s(x), y 1 s(0)=1 \\
v 1 s^{\prime}(x)=-\omega^{2}\left(1-w \cdot \operatorname{sign}(y 1 s(x) y 2 s(x))^{2} y 1 s(x), v 1 s(0)=0\right. \\
y 2 s^{\prime}(x)=v 2 s(x), y 2 s(x)=0 \\
v 2 s^{\prime}(x)=-\omega^{2} \cdot(1-w \cdot \operatorname{sign}(y 1 s(x) y 2 s(x)))^{2} y 2 s(x), v 2 s(0)=1
\end{array}\right.
$$

Depending on the signature sign the control will provide the damping of one solution and the rising of the other in full accordance with the analysis given in [3]. The control sign alteration $u s(x)=-\operatorname{sign}(y 1 s(x) \cdot y 2 s(x))$ in comparison with (7) is explained by the fact that $\operatorname{sign}\left(y 1^{\prime}(x)\right)=-\operatorname{sign}(y 2(x))$. Returning to the Arnold's analysis we note that the matrix $A$ ' becomes diagonal according to the period definition at the 
feedback introduction: $a_{12}=y 1^{\prime}(T)=a_{21}=y 2(T) \equiv 0$. So, the feedback introduction procedure may be considered as the physical analogy to the mathematical procedure of matrix diagonalization reduced to its eigenvalues and eigenvectors determination.

To finish with this section, we have to illustrate the case when the frequency switching occurs not at doubled frequency but at the fundamental one $(\mathrm{k}=2)$ that is in the points corresponding to maxima or antinodes. It's clear that there's no energy changes in this case (see the previous section), but the oscillations will not be strictly harmonic. That is why the phase trajectory represents the somehow deformed ellipse - the steady limit cycle - figure 4 .

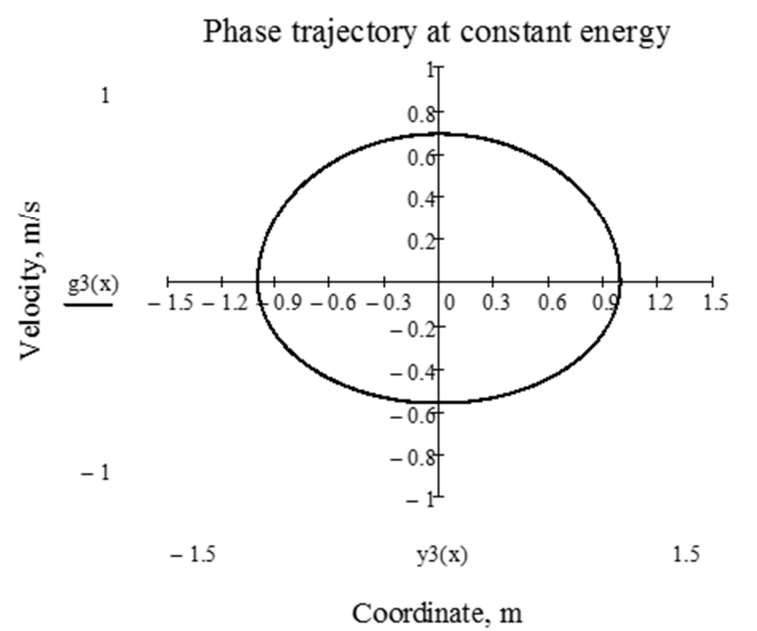

Figure 4. Phase trajectory corresponding to the energy conservation during the period.

The steadiness consists in the fact that any initial conditions changes will not change principally the solution's type but only slightly deform the phase trajectory. Thus, the statement about the parametric resonance existence at $\mathrm{k}=2$ for this kind of modulation is strictly wrong. At the approximate coincidence of the modulation frequency with the oscillation one and switching in the points symmetrical relative to the origin these oscillations are strictly periodic (with doubled control period) due to the energy changes absence during the period.

Due to the importance of this section let us list its main results. The heuristic (based on the energy behavior analysis) feedback introduction in the oscillation equation (at the conjunction conditions execution) permits one to prove that the frequency modulation satisfying the parametric resonance condition is not necessary and sufficient condition of the oscillations unlimited increase. In other words, the statement: "Thus, at $\omega \approx \mathrm{k} / 2, \mathrm{k}=1,2, \ldots$ the lowest position of the idealized swing is extremely unsteady and it shakes up at arbitrary small periodic change of length $[4$, p. 107]" is true not for all periodic laws of the length alteration.

The oscillations damping/shaking up formally corresponds by the frequency and duty ratio to the condition of the equality of optical paths to the quarter-wavelength characteristic of the interference filter or mirror. So the optical-mechanical analogy shows itself not only in the Fermat Principle but the parametric resonance too due to the general Hill's equation description. However, the investigation of light amplification/decay needs the complex wave vector in this equation (see, for instance [13]) which is outside the range of the article. Returning to mechanics one may note that major properties of super lattices hard coatings (see, for instance [14]) are explained by acoustic waves (optical phonons) behavior in them also described by the Hill's equation. Generally, this equation theory underlies most of metamaterials [15] advantages because all transport phenomena imply different wave - electromagnetic, acoustic, spin etc. propagation one way or another.

The difference of solutions types is determined exclusively by the control signature signs. Naturally, the question about the control uniqueness (that is the modulating frequency, duty ratio and signature sign uniqueness) arises. The question about the existence of characteristic index extreme value in different controls is tightly adjoined to the former.

\section{The Optimal Control Theory Application}

The easiest way to answer the questions put in the end of the previous section is to apply the optimal control theory (OCT) [5]. It permits one to receive rather easily damping solutions for piecewise control even for the simplest problem definition - quick-action problem. For such problems the figure of merit is the time needed to hit the phase plane origin. Let's give the similar problem's definition for the $2^{\text {nd }}$ Hill's equation fundamental solution. This problem is defined this way: one needs to find out the control $\mathrm{u}(\mathrm{x})$ which provides the quickest phase plane origin hitting. In the OCT the state equations - are the $1^{\text {st }}$ order ordinary differential equations (ODE) $y_{i}^{\prime}=f_{i}\left(y_{1}, y_{2}, \ldots, y_{r} ; u_{1}, u_{2}, \ldots, u_{k}\right)$ that's why let's rewrite the Hill's equation in the system form using underfoot to differ the $2^{\text {nd }}$ variable from the $2^{\text {nd }}$ fundamental solution:

$$
\left\{\begin{array}{c}
y_{1}^{\prime}(x)=y_{2}(x), \quad y_{1}(0)=0 \\
y_{2}{ }^{\prime}(x)=-\omega_{0}^{2}(1+w \cdot u(x))^{2} \cdot y_{1}(x), \quad y_{2}(0)=1
\end{array}|u(x)| \leq 1\right.
$$

The figure of merit Kopt $=\int_{0}^{X} d x$, so that the zeroth state equation looks like $y_{0}{ }^{\prime}(x)=1, y_{0}(0)=0$. Then the conjugated equations (in OCT terms) are the ODE system:

$$
p_{i}^{\prime}=-\sum_{j=0}^{r} \frac{\partial f_{j}}{\partial y_{i}} p_{j} \Rightarrow\left\{\begin{array}{c}
p_{1}{ }^{\prime}(x)=\omega_{0}^{2}(1+w \cdot u(x))^{2} \cdot p_{2}(x), p_{1}(0)=p_{1,0} \\
p_{2}{ }^{\prime}(x)=-p_{1}(x), p_{2}(0)=p_{2,0}
\end{array}\right.
$$

The optimal control is found from the Pontryagine's maximum principle [5] according to it the optimal control corresponding to the figure of merit minimum corresponds to the "optimal Hamiltonian" maximum. Due to the linear dependence of the "optimal Hamiltonian" $h(x)$ on the control $u(x)$ it's necessary that it changes its sign at the product's $p_{2}(x) y_{1}(x)$ sign alteration to maximize the "optimal Hamiltonian": 


$$
\begin{aligned}
& h(x)=p_{1}(x) y_{2}(x)-\omega_{0}^{2}(1+w \cdot u(x))^{2} p_{2}(x) y_{1}(x)-1 \Rightarrow \\
& u(x)=-\operatorname{sign}\left(y_{1}(x) \cdot p_{2}(x)\right)
\end{aligned}
$$

Besides the maximal value of the optimal Hamiltonian must be zero everywhere that is the state equations vector is normal to the conjugated variables one because the optimal
Hamiltonian is their scalar product:

$$
h(x) \equiv \sum_{i=0}^{r} f_{i}(x) p_{i}(x) \equiv(\vec{f}, \vec{p})=0
$$

However, this condition permits one to determine only $p_{1,0}$ from (13):

$$
h(0)=p_{1}(0) y_{2}(0)-\omega_{0}^{2}(1+w \cdot u(0))^{2} p_{2}(0) y_{1}(0)-1=0 \Rightarrow p_{1}(0) \cdot 1=1 \Rightarrow p_{1,0}=1
$$

The initial speed (derivative) value is not zeroth instead of "heuristic feedback introduction through the fundamental solutions product" but coincides with the initial speed value $y_{2}(0)$ in (12). As for $p_{2,0}$, that is the initial value of the conjugated function itself one may determine only its sign not magnitude. Because at $y_{1}(0)=0$ its sign is positive than the $p_{2,0}$ sign - negative. It follows from the optimal Hamiltonian maximum need at arbitrary small initial value of $y_{1}(x)-$ the control must be negative. Thus, at any negative initial values of $p_{2,0}$ oscillations must damp but the damping efficiency will be naturally different. It's clear that to draw an analogy with the heuristic feedback introduction the initial condition for the conjugated coordinate must be equal to $-\infty$ at the non-zeroth speed value. Therefore, the solutions coincidence - the optimal and heuristic ones in this particular case is possible only in the limit - at the conjugate coordinate initial value going to $-\infty$. But, as it was noted above, at any other negative values an oscillation damping is provided with different efficiency. In figure 5 the solution $-\mathrm{ym}(\mathrm{x})$, control $-\mathrm{um}(\mathrm{x})$ and energy dependence for the $2^{\text {nd }}$ fundamental solution are given (the phase trajectory is similar to the figure 2).

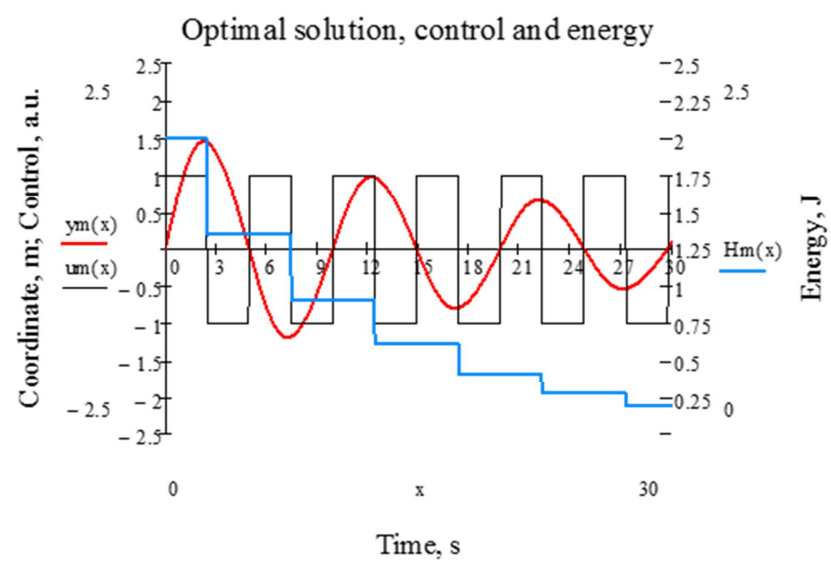

Figure 5. The $2^{\text {nd }}$ fundamental solution (coordinate) at optimal frequency modulation, optimal control and system 's energy.

This solution is obtained for the initial value $p_{2,0}=-10$. Its alteration (unlike the alteration of the initial value at the heuristic feedback introduction) leads to the oscillation frequency, control and duty ratio alteration. Criteria (8) in this particular case are equal: $K m=22,149, S m=6,404$. Let's note that at heuristic feedback introduction the corresponding criteria are equal: $K e=22,231, \quad S e=6,251 ; K s=22,232$, $S s=6,247$. Thus, the optimal control for the initial value $p_{2,0}=$ -10 provides somewhat quicker oscillation damping than the heuristic one but worse from the energy damping viewpoint. The minimal value of the figure of merit KoptM=22,096 corresponds to $p_{2,0} \in-(17,18)$. At $p_{2,0}=-17,5$ the frequency $\omega_{\text {opt }}=0,997 \omega$, and duty ratio $d r_{\text {opt }}=2,088$.

The difference from the heuristic control in this particular case consists of the fact that the frequency switching occurs not in points corresponding to the solution maximum but somewhat later - the delay is equal only to 0.05 of the modulating frequency half-period. Of course, at the modulation depth rise this difference rises too. The oscillation damping improvement in the sense of the criterion introduced above at the frequency switching not strictly in antinodes (whereas strictly in nodes) is explained rather simply. Although the maximal energy decrease and correspondingly the oscillation amplitude really occurs at the frequency switching strictly in nodes, the motion time with smaller frequency is greater than at optimal control. So, the quickest oscillation damping occurs when the resulting frequency and duty ratio become somewhat smaller than the heuristic ones, though only a little at the small modulation depth.

\section{The Mixed Hamiltonian}

Let's note the similarity of the optimal control problem solution and the one obtained at the heuristic feedback introduction through fundamental solutions product. The "shorten" that is not containing -1 optimal Hamiltonian coincides (with the accuracy to the sign between the summands) with the quadratic form produced by the problem (11) solutions (see, though, the Appendix 6 from [4]):

$$
H s(x)=v 1 s(x) \cdot v 2 s(x)+\omega_{0}^{2}(1-w \cdot \operatorname{sign}(y 1 s(x) y 2 s(x)))^{2} y 1 s(x) y 2 s(x)
$$

Let's name the (15) form the mixed or general Hamiltonian (in contrast to the optimal Hamiltonian). Its equality to zero for simple harmonic oscillations is checked immediately. It is zero as the optimal Hamiltonian almost everywhere apart from the zero measure set that is switching points. At the introduction of state (for the $1^{\text {st }}$ fundamental solution and its derivative) and conjugate (for the $2^{\text {nd }}$ fundamental solution and its derivative) equations vectors one can say that they are normal to each other.

Now let's note the difference in conjugate equations type. 
For the heuristic feedback introduction they are strictly the same as the state equations (12) (due to the differential form self-conjugation), only initial values differ. At that only the equality to zero of the "conjugated" function and its derivative sign initial value are important, in the case (12) - it is "+", whereas this value itself may be arbitrary, so that in this case the "conjugated" solution is determined with the accuracy of any positive multiplicative constant. Any change of constant doesn't lead to the control change and correspondingly the "state equations system solution". The conjugated equations of the optimal control problem differ by signs from the state equations that is instead of speed the function opposite in sign to it is introduced. Correspondingly in the speed equation the sign "+" stands in the right part, so that differentiating this equation (in the interval of constant control) and the speed substitution one gets the ordinary oscillation equation.

Therefore, the conjugated variables of the optimal control problem describe exactly the same oscillating system as the investigated one described by the state equations but with other initial conditions. Moreover, at the "shorten Hamiltonian" use the conjugated equations solution exactly corresponds to the $1^{\text {st }}$ fundamental solution, the only difference consists of the speed determination (its sign) and consequently the Hamiltonian - by analogy in sign bounding the "kinetic" and "potential" energies. If one uses the full optimal Hamiltonian the main difference consists in the initial values of the conjugated variables.
So, in the OCT the optimal Hamiltonian equality to zero determines the control. At the heuristic control determination by the feedback introduction through the fundamental solutions product the equality to zero of the analogous form is the solution conclusion. However, the mixed Hamiltonian introduction permits one to obtain the needed solution from the condition of its minimum (instead of Pontryagine's maximum principle). In fact from (15) follows that if solutions differ only by the phase shift than the mixed Hamiltonian changes from the doubled energy at zero shift to zero at the shift equal to $\pi / 2$. (Damping/shaking up with different efficiency oscillations correspond to intermediate phase values.)

The availability of explicit dependency upon time in a motion equation may be considered as the result of "external influence" on the described system. If one considers these influences as controls than the optimal control or feedback introduction automizes systems. At that the conjugated systems energies are not conserved but the mixed Hamiltonian as well as the optimal one is constant (almost everywhere) and equal to zero. Its constancy reflects the fact of the conjugated systems autonomies and piecewise control type whereas energy is periodically added to one system and subtracted from the other. But due to the constancy and equality to zero of the mixed Hamiltonian together with the Wronskian these energies product is the periodic piecewise function and its averaged value upon the modulation period is strictly constant:

$$
\begin{gathered}
H 1(x) \cdot H 2(x)=\frac{1}{4}\left\{(W(x))^{2} \omega^{2} \cdot(1+w \cdot u s(x))^{2}+(H s(x))^{2}\right\}=\frac{1}{4} W^{2} \cdot \omega^{2}(x), \\
\langle H 1(x) \cdot H 2(x)\rangle=\frac{1}{T_{\Sigma}} \int_{0}^{T_{\Sigma}} H 1(x) H 2(x) d x=\frac{1}{4} W^{2} \cdot \omega^{2} \cdot\left(1-w^{2}\right)
\end{gathered}
$$

The other consequence of the mixed Hamiltonian equality to zero together with the Wronskian constancy is the useful analogous in form to the Liouville's theorem equation:

$$
W \cdot \frac{d}{d x}(y 1(x)+y 2(x))=2(y 1(x) \cdot H 2(x)-y 2(x) \cdot H 1(x))=2[y(x), H(x)],
$$

where the commutator is denoted by brackets as usually whereas $H 1(x)$ and $H 2(x)$ - are ordinary Hamiltonians of conjugated equations (systems). The particularly simple form (17) has at the Wronskian equality to unity whereas for simple harmonic oscillations $(W=\omega)$ the equality (17) is checked immediately.

\section{The Control at an Arbitrary Phase Shift}

From the previous section results follows that the damping/shaking up efficiency is determined in particular by the phase shift between the solutions whose product is chosen as the control "key". To estimate this shift influence let's generalize the (10) formulae on the case of the switching in an arbitrary point accounting the conjugation conditions (6). Let the $1^{\text {st }}$ switching occur in the point corresponding to the initial phase $\psi \in(-\pi / 2, \pi / 2)$. From (6) we immediately get the phase of the conjugated function, its period and characteristic index. Let's note that the switching occurs twice during a period. At the $1^{\text {st }}$ one the oscillations amplitudes ratio is equal to the amplitude $B(\psi)$ whereas at the $2^{\text {nd }}$ - to the frequency's ratio as in the (10). Hence, these values must be multiplied at the characteristic index calculation: 


$$
\begin{aligned}
& \phi(\psi)=\frac{1}{\omega(1-w)}\left\{\operatorname{arctg}\left[\frac{1+w}{1-w} \cdot \operatorname{tg}(\psi)\right]-\frac{1-w}{1+w} \cdot \psi\right\} \\
& T(\psi)=\frac{\pi}{\omega \cdot\left(1-w^{2}\right)}-\phi(\psi), \quad B(\psi)=\frac{\cos (\psi)}{\cos \left[\omega(1-w) \cdot\left(\frac{\psi}{\omega(1+w)}+\phi(\psi)\right)\right]} \\
& \alpha(\psi)=\frac{1}{T(\psi)} \cdot \ln \left(B(\psi) \cdot \frac{1-w}{1+w}\right)
\end{aligned}
$$

It's seen that at zero initial phase (18) reduces to (10) with the accuracy to the 2 in the nominator because (10) have been obtained for the half of a period. Characteristic indexes dependencies upon initial phase for three modulation depth values: $w 1=0.1, w 2=0.2, w 3=0.3$ are shown on figure 6 .

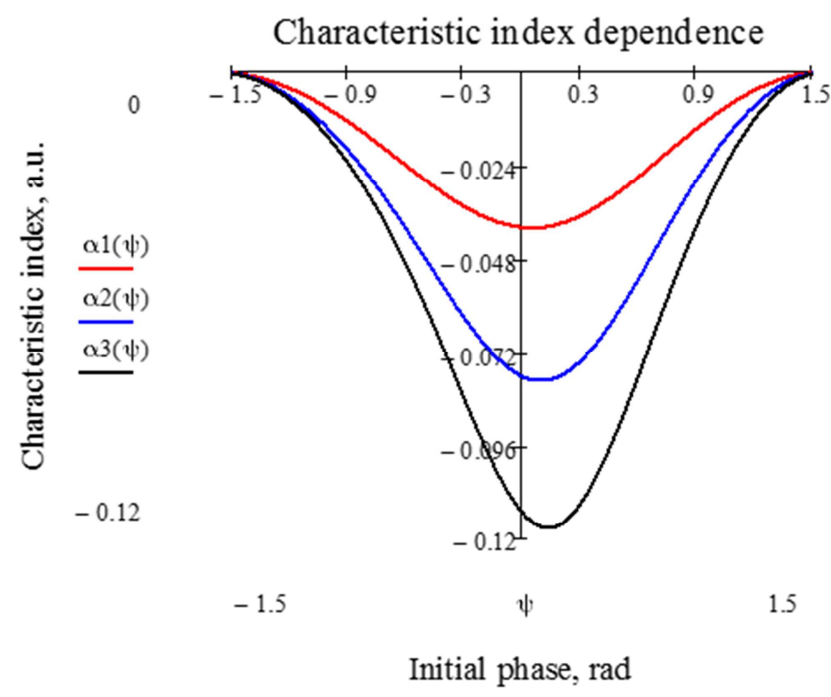

It's seen that as it follows from the OCT application results characteristic indexes reach their minimal values not in the point with minimal initial phase but in the points determined by the modulation depth. Their difference from calculated according to (10) is not large: for the minimal modulation depth and the given calculation accuracy there is no difference at all; for the value 0.2 the index is -0.078 that is less on one thousandth, at last for the modulation depth equal to 0.3 the calculated value is equal to -0.113 that is less on 0.004 .

However, if one takes the obtained in the previous section modulation depth which provides the minimal characteristic index value -0.179 and finds its minimum as the initial phase function one gets already -0.231 for the initial phase 0.22 . Let's note that the significant initial phase introduction transforms the index from a nonmonotonic function into the monotonic one right up to $w=0.99$. At that this modulation depth corresponds to the characteristic index minimal value equal to $-0,305$ at the initial phase equal to 0.24 or $13^{\circ} 45^{\prime}$. This initial phase provides the maximal increase/decrease at a large modulation depth (beginning from around 0.4) which is seen on the figure 7 plots for different initial phases: $0(\alpha)$, $0.1(\alpha 1), 0.24(\alpha 2)$ and $0.35(\alpha 3)$.

Figure 6. The characteristic indexes dependencies on the initial phase at different modulation depths $(w 1=0.1, w 2=0.2, w 3=0.3)$.

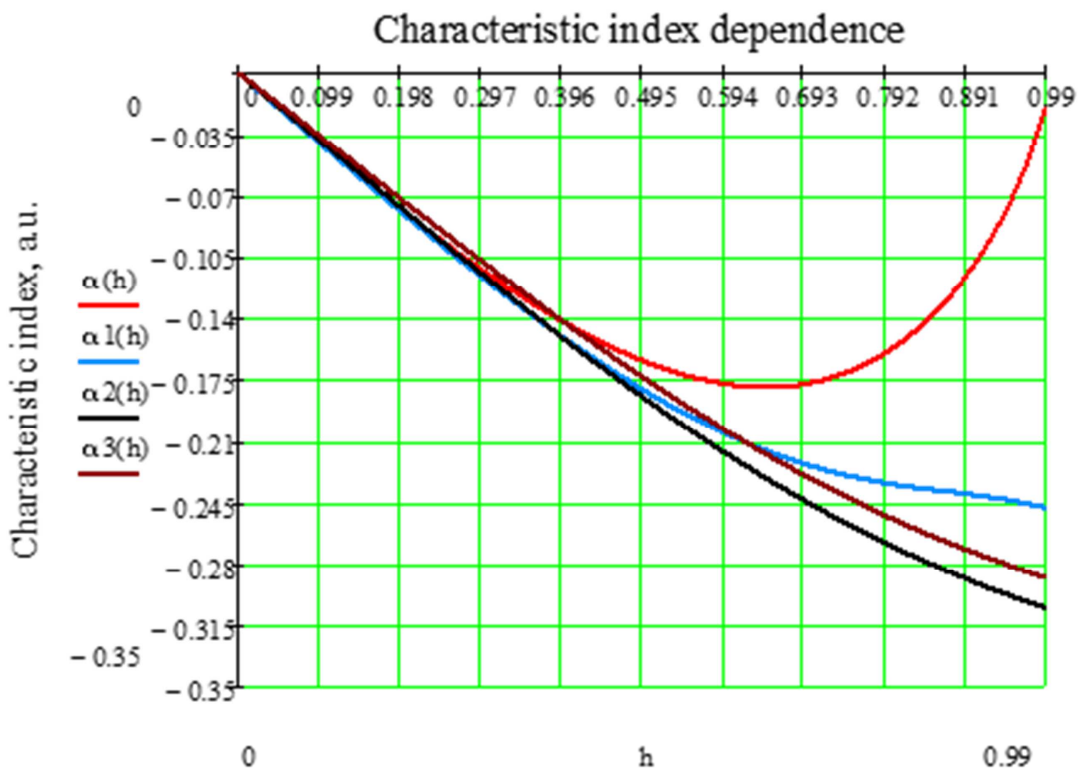

Modulation depth, au.

Figure 7. The characteristic indexes dependencies on the modulation depth for different initial phases. 
From formula (16) one can easily get analytic expressions for the true oscillation frequency and duty ratio as the modulation depth and initial phase functions. The duty ratio versus the initial phase for different modulation depth value plots are presented on figure 8 .

It's interesting for technical applications to determine the control providing the oscillation damping at duty ratio equal to 2 and predetermined modulation depth, say $w 1=0.1$, for example, for the first fundamental solution. To make it one has to determine the initial phase from the duty ratio dependence on the initial phase (fig. 4). At this initial phase one calculates the true oscillation frequency (the reduced shift of this frequency from the fundamental one $\delta \omega(\psi, w)=\omega_{0}^{-1}\left(\omega(\psi, w)-\omega_{0}\right)$ is on the figure 9) and the characteristic (damping) index (figure 6). Now it lasts only to determine the control initial phase providing damping. If the control is presumed piecewise then the initial control phase $\chi$ is found from the condition of the argument equality to zero in the point corresponding to the solution initial phase.

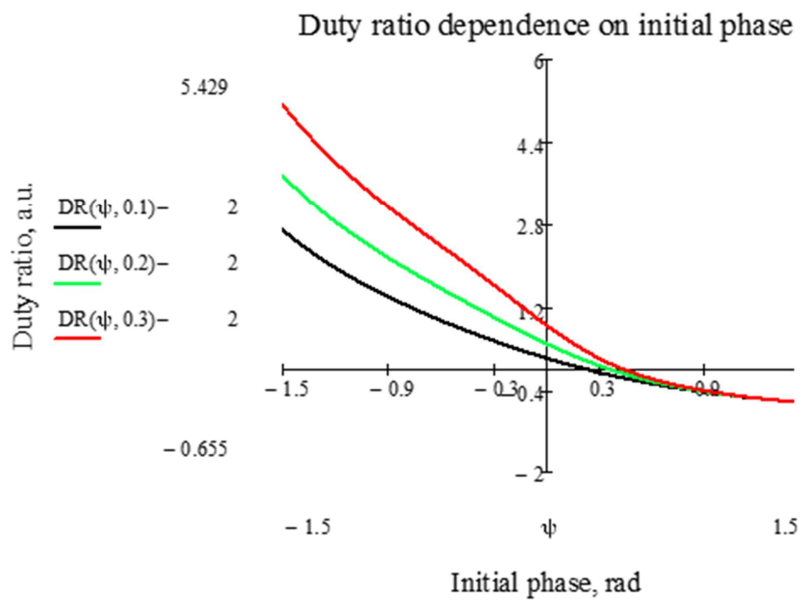

Figure 8. The duty ratio dependence on the initial phase for different modulation depths.

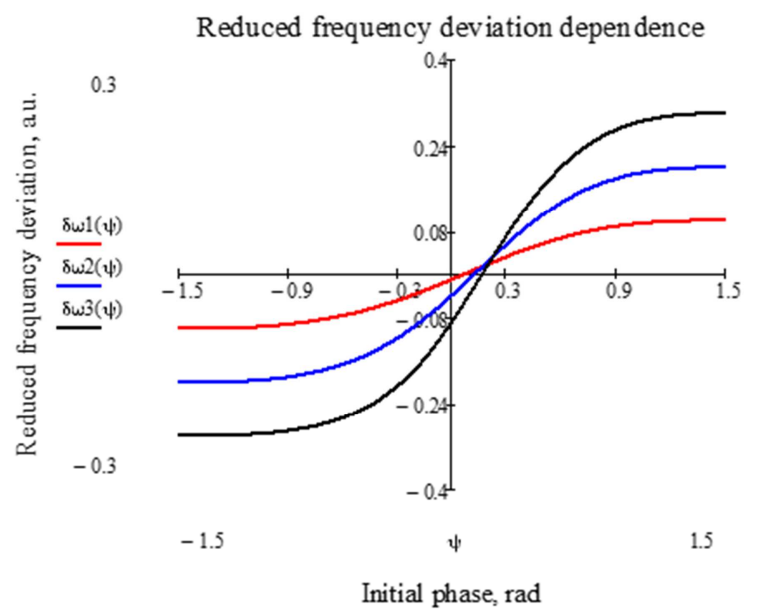

Figure 9. The reduced shift of the true oscillation frequency (from the initial one) $\delta \omega(\psi, w)=\omega_{0}^{-1}\left(\omega(\psi, w)-\omega_{0}\right)$ dependency on the initial phase for different modulation depths.
Thus the control with duty ratio equal to 2 providing oscillation damping for the $1^{\text {st }}$ fundamental solution looks like: $u(t)=-w 1 \cdot \operatorname{sign}[\sin (2,0466 \cdot \omega \cdot t-0,44)]$. The Hill's equation solution $(\mathrm{z}(\mathrm{t}))$ with this control and initial conditions corresponding to the $1^{\text {st }}$ fundamental solution and the approximating function $L 1(\mathrm{t})$ plots are given on the figure 10 . At last the smooth control providing damping differs from the piecewise one by its modulation frequency, initial phase and damping index. These differences for the small modulation depth are not significant but cannot be calculated as easily as in the case of piecewise modulation. For the modulation depth equal to 0.1 the modulation frequency is equal to $2.04239 \omega$ whereas the control initial phase -0.504 . At that the damping index modulo reduces to 0.03 . Hence, to parametric oscillation increase/decrease with the help of smooth modulation with the duty ratio equal to 2 it's enough to correctly choose the modulating frequency and initial phase depending on the control parameter modulation depth. At that the (2) condition of the parametric resonance initiation is not necessary and either sufficient.

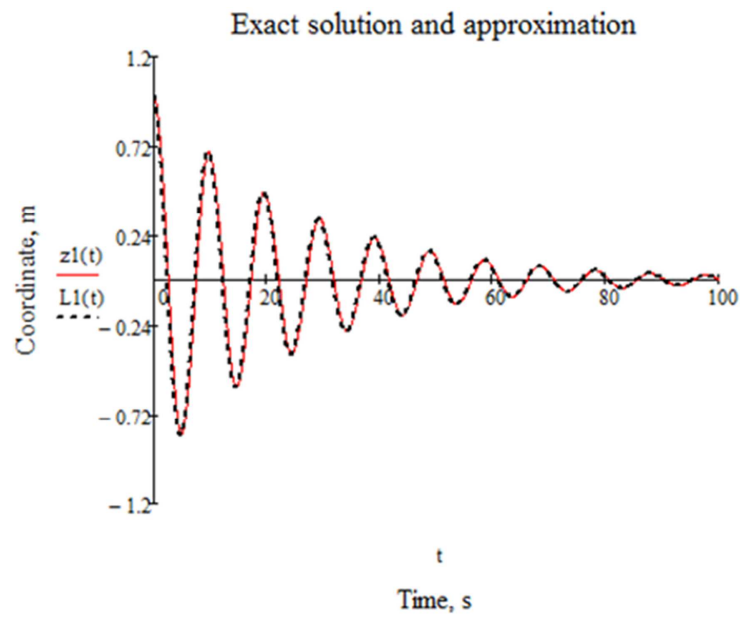

Figure 10. The Hill's controlled equation $1^{\text {st }}$ fundamental solution and its approximating function plots.

\section{The Nonlinearity Accounting}

The simple dependences presented in the previous section seem to exclude the necessity of OCT application, though it's not true. In the general case, for example at any friction existence, the conjugated equation must contain the increase factor due to the sign change of the $1^{\text {st }}$ derivative in the linear differential form. But if this form is not linear the construction of the conjugated one without OCT is hardly possible. Let's consider the general homogeneous differential form interesting for physical application - the motion equation in the form:

$$
L(y)=y^{\prime \prime}+F\left(y, y^{\prime}, x\right)=0 \Rightarrow y^{\prime \prime}=-F\left(y, y^{\prime}, x\right) .
$$

The conjugated differential form may be found with the help of OCT algorithm: 


$$
y_{1}{ }^{\prime}=y_{2}=f_{1}, y_{2}{ }^{\prime}=-F\left(y_{1}, y_{2}, x\right)=f_{2} \Rightarrow p_{1}{ }^{\prime}=\frac{\partial F}{\partial y_{1}} p_{2}, p_{2}{ }^{\prime}=-p_{1}+\frac{\partial F}{\partial y_{2}} p_{2} .
$$

Differentiating as usually the $2^{\text {nd }}$ conjugated solution upon $x$ and substituting the $p_{1}$ ' value from the $1^{\text {st }}$ one we get the conjugated to (8) homogeneous differential form looking like (substituting $p_{2}$ simply onto $-p$ ):

$$
L^{*}(p)=p^{\prime \prime}-\frac{\partial F}{\partial y^{\prime}} p^{\prime}+\left(\frac{\partial F}{\partial y}-\frac{\partial^{2} F}{\partial y^{\prime} \partial x}\right) p=0 \Rightarrow p^{\prime \prime}=\frac{\partial F}{\partial y^{\prime}} p^{\prime}-\left(\frac{\partial F}{\partial y}-\frac{\partial^{2} F}{\partial y^{\prime} \partial x}\right) p
$$

This equality (10) is immediately checked for the case of linear differential form (8), for example, at the friction availability. It's seen that in the general case the conjugated form depends upon the solution of the main one - only linear forms are independent. The mixed and optimal Hamiltonians and canonic equations look like (compare with $(8,9))$ :

$$
\begin{aligned}
& H s=y^{\prime} p^{\prime}+p\left(F\left(y, y^{\prime}, x\right)-y^{\prime} \frac{\partial F\left(y, y^{\prime}, x\right)}{\partial y^{\prime}}\right)=y_{2} \cdot p_{1}-F\left(y_{1}, y_{2}, x\right) p_{2}=\text { Hopt } \Rightarrow \\
& \frac{d}{d x} y=y_{1}^{\prime}=\frac{\partial H o p t}{\partial p_{1}}=y_{2}, \frac{d}{d x} y^{\prime}=y_{2}{ }^{\prime}=\frac{\partial H o p t}{\partial p_{2}}=-F\left(y_{1}, y_{2}, x\right), \\
& p_{1}^{\prime}=-\frac{\partial H o p t}{\partial y_{1}}=\frac{\partial F}{\partial y_{1}} p_{2}, p_{2}^{\prime}=-\frac{\partial H o p t}{\partial y_{2}}=-p_{1}+\frac{\partial F}{\partial y_{2}} p_{2}
\end{aligned}
$$

For example the physical pendulum oscillation equation looks like [3]:

$$
\begin{aligned}
& y^{\prime \prime}+\omega^{2}(x) \cdot \operatorname{Sin}(y)=0 \Rightarrow H s=y^{\prime} p^{\prime}+\omega^{2}(x) \operatorname{Sin}(y) p \Rightarrow \\
& p^{\prime \prime}=-\frac{\partial H s}{\partial y} p=-\omega^{2}(x) \operatorname{Cos}(y) p
\end{aligned}
$$

In the case of constant frequency (autonomic system) numerical solution proves the mixed Hamiltonian constancy and equality to zero. The optimal control is determined now by the signature of the basic and conjugated solutions product. In the case of small oscillations, the control corresponds to the linear oscillation case considered above $(\operatorname{Sin}(y) \approx y, \operatorname{Cos}(y) \approx 1)$. At the time-depending frequency substitution by the control function the mixed Hamiltonian immediately becomes zero as well as the optimal one. Other nonlinearities may be accounted in the similar way.

\section{Conclusion}

The parametric resonance analysis given in [2-4] being true in general is though incomplete and strict. It follows from the above consideration that:

- there exist an infinite set of modulating frequencies and duty ratios satisfying the parametric resonance initiation condition and providing oscillation damping/rise up at the feedback introduction through the product of solutions differing by their initial phase;

- at that the solutions are conjugated that is at phase shift corresponding to $\pi / 2$ the bilinear form (15) - the mixed (general) Hamiltonian analogous to the optimal one is zero almost everywhere too, the one's system oscillations are damping whereas the other's - conjugated - are rising up;

- at the absence of phase shift no frequency modulation occurs because the solution square doesn't change its sign and oscillations are strictly harmonic with maximal or minimal frequency depending on the signature sign;

- for a given modulation depth there exist the optimal phase shift providing the quickest oscillation damping and strictly determined frequency and duty ratio correspond to it, their knowledge permits one to implement explicitly the control without any feedback introduction.

This feedback introduction is possible either by the piecewise - signatures - or smooth functions, though piecewise control provides the best oscillation decrease/increase in the sense of the introduced figures of merit and the mixed Hamiltonian equality to zero almost everywhere. The feedback in the form of fundamental solutions product signature clearly illustrates the physical sense of the optimal control problem's conjugated variables and optimal Hamiltonian on the Hill's equation solutions class.

\section{References}

[1] "Differentialgleichungen Lösungsmethoden und Lösungen" von Dr. E. Kamke, I, Gewöhnliche Differentialgleichungen, 6. Verbesserte Auflage, Leipzig, 1959.

[2] Berezkin E. N. The theoretical mechanics course, Moscow, Moscow State University publishing house, 1974 (in Russian), $646 \mathrm{p}$.

[3] Landau L. D., Lifshits The theoretical physics short course, book 1: Mechanics. Electrodynamics, Moscow, "Nauka", 1962 (in Russian), 272 p.

[4] Arnold V. I. The mathematical methods of the classic mechanics, Moscow, "Nauka", 1979 (in Russian), 475 p.

[5] Pontryagin L. S., Boltyanskiy V. G., Gamkrelidze R. V., Mischenko E. F. The mathematical theory of optimal processes, Moscow, "Nauka”, 1983 (in Russian), 392 p.

[6] Vainstein L. A., Vakman D. E. The frequencies division in the vibrations and waves theory, Moscow, "Nauka", 1983 (in Russian). 
[7] Aleshkevitch V. A., Dedenko L. G., Karavaev V. A. Oscillations and Waves. Lectures. (The General Physics University Course). - Moscow, MSU Physics Faculty, 2001 (in Russian), $144 \mathrm{p}$

[8] Vyatchanin S. P. Radio-physics lecture notes. http://hbar.msu.ru (in Russian)

[9] Butikov E. I. Parametric Resonance KIO http://butikov.faculty.ifmo.ru (in Russian)

[10] Yablonskiy A. A., Noreiko C. C. The Oscillation Theory Course, Sankt-Petersburg, "Lan M", 2003 (in Russian)

[11] Trubetskov D. I., Hramov A. E. Lectures on microwave electronics for physicists. N 2, V. 1, Moscow, PHYSMATLIT, 2003, (in Russian), 496 p.

[12] Parshakov A. N. The oscillations physics: school-book/ A. N. Parshakov. Perm State University publishing house, 2010 (in Russian), 302 p.

[13] H. Kogelnik, C. V. Shank, J. Appl. Phys. 43, 1972, 2327.

[14] J. Musil, Hard and superhard nanocomposite coatings // Surf. Coat. Technol., 2000, 125, 322-330.

[15] Rodger M. Walser, Electromagnetic Metamaterials. Inaugural Lecture // Proceedings of SPIE Vol. 4467 (2001), P. 1-15 (C) 2001 SPIE. 